\section{7}

9

1

2

\title{
Soft Tissues Store and Return Mechanical Energy in
} Human Running

Riddick, RC, Kuo, AD.

Ryan Riddick: riddryan@umich.edu,240-688-1708

Art Kuo:artkuo@umich.edu,734-764-1817

Affiliations: University of Michigan

Address: G.G. Brown Laboratory, 2350 Hayward St., Ann Arbor MI 48109

Keywords: mechanical work, soft tissues, energetics, deformation, metabolic cost

Word count: 3483

8

0

1

(1)

3

4

5

6

7

8

9

\footnotetext{
(C) 2016. This manuscript version is made available under the Elsevier user license http:/www.elsevier.com/open-access/userlicense/1.0/
} 
Abstract:

34 During human running, softer parts of the body may deform under load and dissipate mechanical energy.

35 Although tissues such as the heel pad have been characterized individually, the aggregate work performed

36 by all soft tissues during running is unknown. We therefore estimated the work performed by soft tissues

$37(\mathrm{~N}=8$ healthy adults $)$ at running speeds ranging $2-5 \mathrm{~m} \cdot \mathrm{s}^{-1}$, computed as the difference between joint

38 work performed on rigid segments, and whole-body estimates of work performed on the (non-rigid) body

39 center of mass (COM) and peripheral to the COM. Soft tissues performed aggregate negative work, with

40 magnitude increasing linearly with speed. The amount was about $-19 \mathrm{~J}$ per stance phase at a nominal 3

$41 \mathrm{~m} \cdot \mathrm{s}^{-1}$, accounting for more than $25 \%$ of stance phase negative work performed by the entire body.

42 Fluctuations in soft tissue mechanical power over time resembled a damped oscillation starting at ground

43 contact, with peak negative power comparable to that for the knee joint (about $-500 \mathrm{~W}$ ). Even the positive

44 work from soft tissue rebound was significant, about $13 \mathrm{~J}$ per stance phase (about 17\% of the positive

45 work of the entire body). Assuming that the net dissipative work is offset by an equal amount of active,

46 positive muscle work performed at $25 \%$ efficiency, soft tissue dissipation could account for about $29 \%$ of

47 the net metabolic expenditure for running at $5 \mathrm{~m} \cdot \mathrm{s}^{-1}$. During running, soft tissue deformations dissipate

48 mechanical energy that must be offset by active muscle work at non-negligible metabolic cost.

\section{Introduction}

53 During steady-state running, the body performs active and passive work summing to zero over each

54 stride. As characterized previously (Farris and Sawicki, 2011; Novacheck, 1998; Schache et al., 2011;

55 Winter, 1983), the leg joints perform positive and negative work within each stride, with active muscles

56 accounting for nearly all of the metabolic energy expenditure (Margaria et al., 1963). Passive "soft

57 tissues" such as the heel pad (Chi and Schmitt, 2005), cartilage, and intervertebral discs (among others, 
58 see Fig. 1) help cushion the body. That cushioning functions in part to dissipate mechanical energy.

59 Although their mechanical properties are well understood, it is unknown how much energy soft tissues

60 dissipate in aggregate and in comparison to muscles. An estimate of soft tissue work might provide

61 insight on the active work of muscles and the attendant metabolic cost of running.

62 The leg joints account for most of the mechanical power produced during running. Although the joint

63 power trajectories are rather complex, their effect on the whole body is fairly simple: Following a brief

64 small burst of positive work (about $0-2.5 \%$ of a stride starting at ground contact), most of the stance

65 phase resembles a mass-spring system (McMahon and Cheng, 1990), with one main large burst of

66 negative work (2.5 - 15\% stride, termed Collision here), and then one large burst of positive work (15-

$6734 \%$ stride, termed Rebound) prior to the Aerial (34-50\%) and Swing (50-100\%) phases. Much of the

68 work observed at the joints might actually be performed by elastic tendon. The amount is measured to be

69 more than half in turkeys (Roberts et al., 1997), and indirectly projected to be similar in humans (Cavagna

70 and Kaneko, 1977). If there were no other dissipation in the body, then the work fluctuations would be

71 due only to elastic tendon and to equal magnitudes of active negative and positive work by muscle, all

72 summing to zero over a stride.

73 There is, however additional dissipation by soft tissues. The heel pad, plantar fascia, and other elements

74 of the foot both cushion the body and provide elastic energy return (Ker et al., 1988). Soft tissues in the

75 lower leg explain how impact forces are transmitted and damped during running and jumping (Pain and

76 Challis, 2006) and how they can modulate peak joint forces (Liu and Nigg, 2000; Nigg and Liu, 1999;

77 Wakeling and Nigg, 2001). From measurements of skin motion, the thigh and shank also absorb

78 considerable mechanical energy during each stance phase (Schmitt and Günther, 2011). But soft tissue

79 deformations can be challenging to measure, for example through embedded force recordings in cadaveric

80 specimens (Ker et al., 1987; Pai and Ledoux, 2010), or extensive motion capture of the skin (Günther et

81 al., 2003) and potentially for the entire body. As a result, there are no estimates for the overall work 
82 performed by soft tissue deformations during in situ running, nor of the active work needed to offset the

83 dissipation.

84 The aggregate work of soft tissues can be estimated, albeit indirectly. This relies on the discrepancy of

85 total mechanical work performed according to two different measures: (1) joint powers as estimated by

86 rigid-body inverse dynamics, and (2) rate of work performed on the (non-rigid) body center of mass

$87(\mathrm{COM})$ as measured by ground reaction forces, and on the periphery as measured by inverse dynamics

88 (Zelik and Kuo, 2012). Their difference indicates work performed by soft tissues, showing substantial

89 dissipation during human walking (Zelik and Kuo, 2010) and particularly in the obese (Fu et al., 2014).

90 The dissipation is also highly dependent on impacts, as demonstrated in jumping (Zelik and Kuo, 2012),

91 suggesting possible contributions to running as well.

92 Because the muscles must actively offset any net dissipation in the body, soft tissue deformations may

93 affect the metabolic cost of both walking and running. The purpose of the present study was to estimate

94 these contributions during human running. We expect that (1) soft tissues perform both negative and

95 positive work within each stance phase, (2) yielding substantial net negative work, and (3) in increasing

96 amount at faster running speeds, due to the greater impacts.

97

98 Materials and Methods

99 We estimated the work of soft-tissue deformations for 8 young healthy adults (7 males, 1 female; aged 20

$100-34$ yrs.; body mass $M 74.9 \pm 13.0 \mathrm{~kg}$, and leg length $L 0.94 \pm 0.044 \mathrm{~m}$, mean \pm s.d.) running at a range of

101 speeds. The subjects ran on an instrumented treadmill at a comfortable range of speeds determined by

102 their level of fitness (a total range of 2.2 to $5 \mathrm{~m}^{\cdot-1} \mathrm{~s}^{-1}$ ) in 7 randomized experimental trials lasting 1 minute

103 each. Of the 8 subjects, 6 landed on their rear-foot, while two tended to land on their mid-foot or fore-

104 foot, as determined by observing the subjects' gait. All subjects gave informed consent according to

105 approved Institutional Review Board procedures. 
We recorded ground reaction forces and motion capture. Forces were recorded on a split-belt instrumented treadmill (Bertec, Columbus, OH, USA) at $960 \mathrm{~Hz}$. Kinematic data were collected at 480 Hz with an eight-camera motion capture system (PhaseSpace Inc., San Leandro, CA, USA), with markers

109 placed bilaterally on the ankle (lateral mallelous), knee (lateral epicondyle), hip (greater trochanter),

110 shoulder (acromion of scapula), elbow (lateral epicondyle of humerus), and wrist (trapezium). Additional

111 tracking markers were placed on the shanks, thighs, trunk, upper and lower arm, pelvis (sacrum, left/right

112 anterior superior iliac spine) and each foot (calcaneous, fifth metatarsal). Inverse dynamics calculations

113 (Fig.2) were performed using standard commercial software (Visual3D, C-Motion, Germantown, MD,

114 USA) and its associated anthropometric model with 6-DOF joints. Analog force data were filtered at 25

$115 \mathrm{~Hz}$ and marker motion was filtered at $10 \mathrm{~Hz}$ (low-pass Butterworth) before computing inverse dynamics.

116 These data were used to estimate soft tissue work using a previously developed method (e.g., (Fu et al.,

117 2014; Zelik and Kuo, 2012)). At each point in time during a stride, the joint powers derived from rigid

118 body inverse dynamics, and the unknown soft tissue power, must sum to equal the whole body

119 fluctuations in mechanical power ("total mechanical power"). For the former, we use the term Joint

120 Power for the aggregate power from all measured joints (and originating from positive and negative work

121 of muscles and tendons) using inverse dynamics, summed at each sample in time (Fig. 3A). Joint Power

122 cannot differentiate between possible sources of the power, which could include muscle, tendon, friction,

123 and other deformations. Individual joint powers were computed from relative three-dimensional rotation

124 and translation of each connected pair of body segments (also called "6-DOF powers"), which helps

125 account for inaccurate joint center locations (Buczek et al., 1994; Fu et al., 2014).

126 For total mechanical power, we computed the sum of work rates on the COM (Donelan et al., 2002) and

127 for moving body segments relative to the COM (termed COM work rate and peripheral work rate or

128 power, respectively). The COM work rate was calculated (Fig. 3B) from the three-dimensional dot

129 product of ground reaction force and the COM velocity, the latter estimated by integrating forces

130 assuming periodic strides. Peripheral power (Fig. 3B) to move segments relative to the COM was 
131 calculated as the time derivative of translational and rotational energy of segments relative to the COM

132 (Cavagna and Kaneko, 1977; Zelik and Kuo, 2012). While COM work includes soft tissue motions,

133 peripheral power relies on rigid body kinematics, and therefore neglects soft tissue deformations.

134 We used the difference between Total mechanical power and Joint Power as an indicator of soft-tissue 135 work (Figs. 3C \& 4). We expected considerable soft tissue negative work during Collision, with some 136 fraction returned elastically as positive work, but with net negative work over a stride. This also implies 137 that Joint Power might yield net positive work over a stride, as is the case for walking (Kuo et al., 2005; 138 Zelik and Kuo, 2012). To summarize the work over a stride, we computed the positive, negative, and net 139 work for each power quantity, for example by integrating only the positive intervals within a stride to 140 yield positive work per stride. Corresponding average work rates or powers were computed by

141 multiplying work per stride by stride frequency.

142 Analyses were normalized as follows. Joint moments, angular velocities, and powers were averaged 143 across multiple strides as a function of stride time $(0-100 \%)$ for each subject and condition. To account 144 for differences in subject size, we used body mass $M$, leg length $L$, and gravitational acceleration $g$ as 145 base units for non-dimensionalization. Mean power and work normalization constants were $M g^{3 / 2} L^{1 / 2}=$ $1462184 \mathrm{~W}$ and $M g L=678 \mathrm{~J}$ respectively; mean running speed normalization constant was $g^{1 / 2} L^{1 / 2}=3.04$ $147 \mathrm{~m} \cdot \mathrm{s}^{-1}$.

148 To summarize how work varied with running speed, we used linear regression fits (Cavagna et al., 1977, 149 1964). Regressions were performed using a subject-specific random effects model with constant slope, so 150 that each subject was given a best-fit offset and a slope between the two regression variables that was 151 constant across all subjects. Analysis of covariance (ANCOVA) was used to test for differences between 152 regression coefficients across different measures of mechanical power, with specific comparisons made 153 with post-hoc t-tests. Significance of all statistical tests was tested based on $\alpha=0.05$. To facilitate 154 comparisons, work measures were also reported from regressions at a nominal speed of $3 \mathrm{~m} \cdot \mathrm{s}^{-1}$. 
Results

157 The ankle, knee, and hip were generally observed to produce more power with increasing speed (Fig. 2).

158 Each of these joints had distinct phasing, but with a sum (Joint Power) that resembled a simple sinusoid

159 during stance phase (Fig. 3A). Peripheral work rate was generally found to be out of phase with the work

160 performed on the COM (Fig. 3B). Joint Power qualitatively displayed similar trends of negative and

161 positive work compared to Total work rate during stance phase, with the largest discrepancy occurring at

162 the beginning of collision (Fig. 3C).

163 Soft tissue power exhibited a trajectory over time resembling the damped oscillation of mass-spring-

164 damper system. It began with a large peak of negative power (about $-500 \mathrm{~W}$ at $3 \mathrm{~m} \cdot \mathrm{s}^{-1}$ ), comparable to

165 the peak for the knee (about $-470 \mathrm{~W}$; Fig. 3), followed shortly thereafter by a smaller positive peak of

166 power (Fig. 4). The first peak increased with running speed, whereas the rest of the oscillation amplitude

167 was less clearly dependent on running speed. Both peaks occurred within the Collision phase for the

168 COM, so that the soft tissue oscillation was about twice the frequency of the COM's oscillation.

169 There was an increasing discrepancy between positive and negative joint work with speed. The net

170 summed Joint Work rate was positive and approximately linear with running speed (Fig. 5). In contrast,

171 net Total work rate was close to zero at all running speeds, as expected.

172 Much of the joint work discrepancy was explained by soft tissue work, which was net negative. Net soft

173 tissue power was quite small at low speeds, but became more negative in proportion to speed, accounting

174 for over $20 \%$ of the total negative work of the body at $5 \mathrm{~m} \cdot \mathrm{s}^{-1}$ (Fig. 6 ; see Table 1 for details). The net

175 power was composed of negative power by soft tissues (about $-58 \mathrm{~W}$ at $3 \mathrm{~m} \cdot \mathrm{s}^{-1}$; see Table 1), also

176 changing approximately linearly with speed $\left(-28.5 \mathrm{~W}\right.$ per $\left.1 \mathrm{~m} \cdot \mathrm{s}^{-1}\right)$, as well as a smaller amount of

177 positive work (at about $40 \mathrm{~W}$ rate) not significantly changing with speed (Table 1). The negative power 
from soft tissues was on the same order of magnitude as the negative work of the joints (Fig. 6A; Table

1), particularly the hip (about $-78 \mathrm{~W}$ at $3 \mathrm{~m} \cdot \mathrm{s}^{-1}$ speed).

The magnitudes of negative Total work rate and negative Joint Power increased roughly linearly with

running speed (Fig. 6B). There was a significant difference between the measures in both the slope and

$187 \mathrm{~m} \cdot \mathrm{s}^{-1}$; paired t-tests, $\left.\mathrm{p}=0.58\right)$.

Table 1. Quantitative results for stride parameters and powers, from linear regressions against running speed. Slope and offset refer to regression coefficients ( $\pm 95 \%$ confidence interval, c.i.), reported with , significance ( $\mathrm{S}$ ) denoted by asterisk with criterion $\boldsymbol{P}<0.05$. Dimensionless (d'less) quantities are computed using body mass, leg length, and gravitational acceleration as base units.

\begin{tabular}{|c|c|c|c|c|c|c|c|}
\hline Measure & Normal (SI) & Normal (d'less) & Slope \pm c.i. & Offset \pm s.d. & $\mathrm{R}^{2}$ & $\bar{S}$ & $P$ \\
\hline Speed & $3.07 \pm 0.59 \mathrm{~m} \cdot \mathrm{s}^{-1}$ & $1.01 \pm 0.59$ & $\mathrm{NA}$ & $\mathrm{NA}$ & NA & NA & $\mathrm{NA}$ \\
\hline Step Frequency & $2.81 \pm 0.17 \mathrm{~s}^{-1}$ & $0.87 \pm 0.053$ & $0.15 \pm 0.02$ & $0.72 \pm 0.045$ & 0.85 & * & $8.90 \mathrm{E}-19$ \\
\hline Step Length & $1.09 \pm 0.174 \mathrm{~m}$ & $1.1 \pm 0.18$ & $0.92 \pm 0.036$ & $0.22 \pm 0.056$ & 0.98 & $*$ & $6.20 \mathrm{E}-39$ \\
\hline Stance Time & $0.284 \pm 0.04 \mathrm{~s}$ & $0.91 \pm 0.13$ & $-0.42 \pm 0.042$ & $1.3 \pm 0.07$ & 0.91 & * & $1.00 \mathrm{E}-22$ \\
\hline Aerial Time & $0.0727 \pm 0.0319 \mathrm{~s}$ & $0.23 \pm 0.1$ & $0.22 \pm 0.043$ & $0.00087 \pm 0.08$ & 0.73 & $*$ & $2.60 \mathrm{E}-13$ \\
\hline Pos Total Power & $234 \pm 47.2 \mathrm{~W}$ & $0.1 \pm 0.021$ & $0.071 \pm 0.011$ & $0.033 \pm 0.0096$ & 0.8 & * & $7.80 \mathrm{E}-16$ \\
\hline Pos Joint Power & $235 \pm 55.4 \mathrm{~W}$ & $0.11 \pm 0.025$ & $0.087 \pm 0.014$ & $0.018 \pm 0.013$ & 0.79 & $*$ & $2.50 \mathrm{E}-15$ \\
\hline Pos COM Power & $271 \pm 49.4 \mathrm{~W}$ & $0.12 \pm 0.022$ & $0.099 \pm 0.0052$ & $0.022 \pm 0.0074$ & 0.97 & * & $1.20 \mathrm{E}-33$ \\
\hline Pos Soft Power & $40.3 \pm 23.5 \mathrm{~W}$ & $0.018 \pm 0.011$ & $0.0011 \pm 0.011$ & $0.017 \pm 0.0097$ & 0 & & 0.85 \\
\hline Pos Ankle Power & $150 \pm 34.5 \mathrm{~W}$ & $0.067 \pm 0.015$ & $0.052 \pm 0.017$ & $0.015 \pm 0.014$ & 0.47 & * & $3.70 \mathrm{E}-07$ \\
\hline Pos Knee Power & $83.6 \pm 36.5 \mathrm{~W}$ & $0.037 \pm 0.016$ & $0.015 \pm 0.012$ & $0.022 \pm 0.015$ & 0.13 & $*$ & 0.019 \\
\hline Pos Hip Power & $197 \pm 95.4 \mathrm{~W}$ & $0.088 \pm 0.043$ & $0.15 \pm 0.017$ & $-0.066 \pm 0.021$ & 0.89 & * & $5.60 \mathrm{E}-21$ \\
\hline Neg Total Power & $-236 \pm 53 \mathrm{~W}$ & $-0.11 \pm 0.024$ & $-0.082 \pm 0.011$ & $-0.023 \pm 0.014$ & 0.84 & * & $3.90 \mathrm{E}-18$ \\
\hline Neg Joint Power & $-219 \pm 49.2 \mathrm{~W}$ & $-0.098 \pm 0.022$ & $-0.055 \pm 0.012$ & $-0.041 \pm 0.015$ & 0.66 & * & $2.80 \mathrm{E}-11$ \\
\hline Neg COM Power & $-271 \pm 49.4 \mathrm{~W}$ & $-0.12 \pm 0.022$ & $-0.099 \pm 0.0054$ & $-0.021 \pm 0.0072$ & 0.97 & $*$ & $3.80 \mathrm{E}-33$ \\
\hline Neg Soft Power & $-59.2 \pm 27 \mathrm{~W}$ & $-0.027 \pm 0.012$ & $-0.043 \pm 0.012$ & $0.016 \pm 0.011$ & 0.57 & * & $6.10 \mathrm{E}-09$ \\
\hline Neg Ankle Power & $-104 \pm 29.3 \mathrm{~W}$ & $-0.047 \pm 0.013$ & $-0.039 \pm 0.0077$ & $-0.0072 \pm 0.011$ & 0.72 & $*$ & $7.90 \mathrm{E}-13$ \\
\hline Neg Knee Power & $-218 \pm 64.3 \mathrm{~W}$ & $-0.098 \pm 0.029$ & $-0.12 \pm 0.012$ & $0.025 \pm 0.019$ & 0.92 & * & $6.00 \mathrm{E}-24$ \\
\hline Neg Hip Power & $-81.1 \pm 46.7 \mathrm{~W}$ & $-0.036 \pm 0.021$ & $-0.022 \pm 0.0082$ & $-0.013 \pm 0.019$ & 0.41 & $*$ & $3.20 \mathrm{E}-06$ \\
\hline Net COM Power & $-0.461 \pm 1.65 \mathrm{~W}$ & $-0.00021 \pm 0.00074$ & $-0.00046 \pm 0.0014$ & $0.00019 \pm 0.00048$ & 0.01 & & $5.10 \mathrm{E}-01$ \\
\hline Net Joint Power & $16.9 \pm 43.4 \mathrm{~W}$ & $0.0076 \pm 0.019$ & $0.032 \pm 0.018$ & $-0.024 \pm 0.018$ & 0.23 & * & 0.00099 \\
\hline Net Soft Power & $-18.9 \pm 45.5 \mathrm{~W}$ & $-0.0085 \pm 0.02$ & $-0.042 \pm 0.02$ & $0.033 \pm 0.02$ & 0.31 & * & 0.00011 \\
\hline
\end{tabular}


188 Soft tissue work was most prominent during the stance phase. At nominal $3 \mathrm{~m} \cdot \mathrm{s}^{-1}$, soft tissues accounted

189 for $-18.8 \mathrm{~J}$ of negative work and $12.6 \mathrm{~J}$ of positive work during stance, and practically no work during the 190 aerial phase. Negative soft-tissue work during stance phase accounted for about $27 \%$ of negative Total 191 Work during stance phase (about $-69.0 \mathrm{~J}$ ), and $24 \%$ of negative Total Work during the entire step (about $19277.6 \mathrm{~J})$.

\section{Discussion}

195 We tested if soft tissues contribute significant mechanical work during locomotion and how its magnitude 196 changes with running speeds. Our results show that soft tissues performed significant negative work and

197 positive work during stance phase, with more negative work at faster running speed. We interpret these

198 findings to suggest that soft tissues may behave in aggregate in a damped elastic manner. While the 199 negative work may be helpful for cushioning, the net work has implications for the energetics of running.

200 Moreover, the substantial amount of soft tissue work means that traditional inverse dynamics methods 201 may underestimate the work performed by the body during running, particularly at higher speeds.

202 One of the simplest indicators of soft tissue work is the discrepancy in overall joint work as measured by 203 inverse dynamics. Net Joint Work Rate was positive at about 7\% of the negative Total Work Rate 204 magnitude at $3 \mathrm{~m} \cdot \mathrm{s}^{-1}$, and nearly $18 \%$ at $5 \mathrm{~m} \cdot \mathrm{s}^{-1}$. This discrepancy suggests that joint work fails to 205 capture a portion of the body's negative work that increases with running speed. An additional indicator is 206 the soft tissue estimate, with a net rate of about $-18 \mathrm{~W}$ at $3 \mathrm{~m} \cdot \mathrm{s}^{-1}$ speed. Similar types of discrepancies 207 have previously revealed differences, with rigid-body inverse dynamics failing to explain more of the 208 work required to run downhill rather than uphill (DeVita et al., 2008), to land from a jump compared to 209 taking off (Zelik and Kuo, 2012), or to walk at higher speeds (Zelik and Kuo, 2010) or with obesity (Fu et 
210 al., 2014). Our present finding for level running is consistent with the expectation that greater impacts

211 cause more soft-tissue dissipation.

212 The main effect of soft tissues during running was to perform negative work shortly after ground contact,

213 at about $5 \%$ of a stride (Fig. 3). Its negative power peak was somewhat in advance of the negative work of

214 the joints and COM. This suggests distal contributions, because upper body deformations would be

215 expected to be in phase with the slower oscillation of the COM. In particular, the plantar fascia, heel pads,

216 and tendons in the leg may dominate the negative power peak. The foot and leg appear well-suited to

217 absorb ground impact peaks (Ker et al., 1988), whereas proximal tissues experience smaller impacts (Pain

218 and Challis, 2006; Riemer et al., 2008). For running at $4.8 \mathrm{~m} \cdot \mathrm{s}^{-1}, \mathrm{Schmitt}$ and Günther (2011), estimated

$21930-60 \mathrm{~J}$ dissipated in the thigh and shank, somewhat exceeding our estimates, perhaps because individual

220 soft tissues can potentially oscillate out of phase with each other, and we examine only aggregate effects.

221 Both localized and aggregate measures may help explain soft tissue work and its effect on locomotion.

222 Soft tissue deformations likely also occur elsewhere in the body. In fact, all tissues are technically

223 deformable, although the dissipation should be dominated by the softest tissues in series with the ground.

224 We previously speculated that the intervertebral discs, articular cartilage, and viscera contribute most

225 substantially in walking (Zelik and Kuo, 2010). Here, the earlier timing of soft tissue work relative to

226 COM, suggests that viscera and proximal tissues might perform less mechanical work than an equivalent

227 amount of soft tissue in the leg. In fact, soft tissue appeared to rebound and perform positive work at

228 about the same time that the COM (and therefore viscera and intervertebral discs) was approaching peak

229 negative work. This is consistent with a damped elastic rebound with faster natural oscillation frequency

230 than the overall body. We expect that the point and path of foot-ground contact may influence

231 characteristics of the soft-tissue power such as frequency and magnitude, but have insufficient data to

232 draw conclusions here. 
233 Our findings may have implications for the energetics of running. Humans expend about $900 \mathrm{~W}$ of net 234 metabolic power to run at $3 \mathrm{~m} \cdot \mathrm{s}^{-1}$, and about $1100 \mathrm{~W}$ at $5 \mathrm{~m} \cdot \mathrm{s}^{-1}$ (Margaria et al., 1963). Because steady 235 running requires an average of zero net work, the net negative work of soft tissues must be offset by an 236 equal amount of active positive work by muscle. Assuming an efficiency of 25\% (Margaria et al., 1963), 237 the work to offset soft tissues would account for about $72 \mathrm{~W}$ of metabolic power at $3 \mathrm{~m} \cdot \mathrm{s}^{-1}$ and $319 \mathrm{~W}$ at $2385 \mathrm{~m} \cdot \mathrm{s}^{-1}$, or about $8 \%$ and $29 \%$, respectively, of the net metabolic power at those speeds. Thus, soft tissue 239 dissipation may come at a substantial metabolic cost. But it could also be beneficial for running

240 economy, because without the cushioning of soft tissues, humans might actively cushion themselves more 241 with additional work from muscles (Skinner et al., 2015), with an attendant metabolic cost (Tung et al., 242 2014).

243 One limitation of our soft tissue measure is that it actually captures work not performed at the rigid bodies 244 normally measured during locomotion. Some of our "soft tissues" could therefore actually be considered 245 joints in other methodologies. For example, the longitudinal arch of the foot performs negative and 246 positive work, mediated in part by active muscle (Kelly et al., 2015). The metatarsophalangeal (MTP) 247 joint also performs significant work late in stance during running (over $-20 \mathrm{~J}$ at $4 \mathrm{~m} \cdot \mathrm{s}^{-1}$; Stefanyshyn and 248 Nigg, 1997). We did not record from such joints, whose work would either appear as soft tissue work or 249 be attributed to other measured joints. We also did not measure deformation of bone, which has higher 250 natural frequencies (Reilly and Burstein, 1975) than we could observe and which would be expected to be 251 negligible compared to softer tissues. With higher sampling rates, our methods could theoretically capture 252 aggregate effects of bone deformation. Or with enough motion capture markers, even very small or very 253 rigid deformations could theoretically be measured directly, and treated as virtual joints between smaller 254 and more numerous rigid bodies (Schmitt and Günther, 2011). Many structures in the body could perform 255 significant work not measured by conventional methods of inverse dynamics, but perhaps capturable by 256 more and faster sampling. 
257 Our study was confined to slow to medium running speeds. In that range, soft tissues dissipated an 258 amount of energy proportional to the running speed and returned a constant amount of energy. These

259 linear relationships do not necessarily hold for higher running speeds and sprinting, for which the soft

260 tissue contributions remain unknown. Another limitation was that our estimates use rigid-body models for

261 the work performed peripheral to the COM, therefore not capturing some deformations. Peripheral soft

262 tissue work refers to deformations about the COM that are anti-phase with each other. We expect it to be

263 small, but such motion could therefore potentially add to our own central estimates, although we did not

264 measure it. Finally, by assuming that the net work of steady state running is equal to zero on level ground,

265 we have implicitly assumed that the work of overcoming friction in the air and ground is negligible, an

266 assumption argued for by others (Margaria, 1968; Willems et. Al., 1995).

267 When soft tissues are of interest and expected to play a significant role, standard inverse dynamics may

268 not accurately reflect the energetics of the body. During running, ignoring the energetics of soft tissue is

269 nearly tantamount to ignoring the knee, which had comparable power trajectories (Fig. 3). An

270 understanding of such limitation may be crucial when relying on experimental data to give insight into

271 how humans move or to guide design of locomotive technology.

272 Soft tissues contribute significantly to the dynamics of running in terms of energy dissipation, storage,

273 and return. Their deformations can help cushion the body, performing some of the negative work required

274 of each stance phase and potentially offloading active dissipation by muscle. It can also return some

275 positive work, but most of the negative work must be offset by active positive work by muscle. Besides

276 being important for understanding normal human gait, analysis of soft tissue dynamics may be important

277 for the production of prostheses and robots, which are often built using mostly rigid components. Most

278 biological tissues, and many non-biological materials, deform under heavy loads, with potential

279 consequences for the dynamics of running. 
The authors thank Justin Sung and Daniel Kowalsky for assistance with data collection. This research

was funded in part by the Department of Defense (W81XWH-09-2-0142), Office of Naval Research

(ETOWL), National Institutes of Health (AG0308), and Defense Advanced Research Projects Agency

(M3 Atlas Program).

\section{References}

Buczek, F.L., Kepple, T.M., Siegel, K.L., Stanhope, S.J., 1994. Translational and rotational joint power terms in a six degree-of-freedom model of the normal ankle complex. J. Biomech. 27, 1447-1457. doi:10.1016/0021-9290(94)90194-5

Cavagna, G.A., Heglund, N.C., Taylor, C.R., 1977. Mechanical work in terrestrial locomotion: two basic mechanisms for minimizing energy expenditure. Am. J. Physiol. - Regul. Integr. Comp. Physiol. 233, R243-R261.

Cavagna, G.A., Kaneko, M., 1977. Mechanical work and efficiency in level walking and running. J. Physiol. 268, 467-481.

Cavagna, G.A., Saibene, F.P., Margaria, R., 1964. Mechanical work in running. J. Appl. Physiol. 19, 249-256.

Chi, K.-J., Schmitt, D., 2005. Mechanical energy and effective foot mass during impact loading of walking and running. J. Biomech. 38, 1387-1395. doi:10.1016/j.jbiomech.2004.06.020

DeVita, P., Janshen, L., Rider, P., Solnik, S., Hortobágyi, T., 2008. Muscle work is biased toward energy generation over dissipation in non-level running. J. Biomech. 41, 33543359. doi:10.1016/j.jbiomech.2008.09.024

Donelan, J., Kram, R., Kuo, A.D., 2002. Simultaneous positive and negative external mechanical work in human walking. J. Biomech. 35, 117-124.

Farris, D.J., Sawicki, G.S., 2011. The mechanics and energetics of human walking and running: a joint level perspective. J. R. Soc. Interface rsif20110182. doi:10.1098/rsif.2011.0182

Fu, X.-Y., Zelik, K.E., Board, W.J., Browning, R.C., Kuo, A.D., 2014. Soft Tissue Deformations Contribute to the Mechanics of Walking in Obese Adults: Med. Sci. Sports Exerc. 1. doi:10.1249/MSS.0000000000000554

Günther, M., Sholukha, V.A., Kessler, D., Wank, V., Blickhan, R., 2003. DEALING WITH SKIN MOTION AND WOBBLING MASSES IN INVERSE DYNAMICS. J. Mech. Med. Biol. 03, 309-335. doi:10.1142/S0219519403000831

Kelly, L.A., Lichtwark, G., Cresswell, A.G., 2015. Active regulation of longitudinal arch compression and recoil during walking and running. J. R. Soc. Interface 12, 20141076. doi:10.1098/rsif.2014.1076

Ker, R.F., Alexander, R.M., Bennett, M.B., 1988. Why are mammalian tendons so thick? J. Zool. 216, 309-324. doi:10.1111/j.1469-7998.1988.tb02432.x 
Ker, R.F., Bennett, M.B., Bibby, S.R., Kester, R.C., Alexander, R.M., 1987. The spring in the arch of the human foot. Nature 325, 147-149. doi:10.1038/325147a0

Kuo, A.D., Donelan, J.M., Ruina, A., 2005. Energetic consequences of walking like an inverted pendulum: step-to-step transitions. Exerc. Sport Sci. Rev. 33, 88-97.

Liu, W., Nigg, B.M., 2000. A mechanical model to determine the influence of masses and mass distribution on the impact force during running. J. Biomech. 33, 219-224. doi:10.1016/S0021-9290(99)00151-7

Margaria, R., 1968. Positive and negative work performances and their efficiencies in human locomotion. Int. Z. Für Angew. Physiol. Einschließlich Arbeitsphysiologie 25, 339-351. doi:10.1007/BF00699624

Margaria, R., P. Cerretelli, P. Aghemo, G. Sassi, 1963. Energy cost of running. J. Appl. Physiol. $18,367-370$.

McMahon, T.A., Cheng, G.C., 1990. The mechanics of running: How does stiffness couple with speed? J. Biomech. 23, Supplement 1, 65-78. doi:10.1016/0021-9290(90)90042-2

Nigg, B.M., Liu, W., 1999. The effect of muscle stiffness and damping on simulated impact force peaks during running. J. Biomech. 32, 849-856. doi:10.1016/S0021-9290(99)00048-2

Novacheck, T.F., 1998. The biomechanics of running. Gait Posture 7, 77-95.

Pain, M.T.G., Challis, J.H., 2006. The influence of soft tissue movement on ground reaction forces, joint torques and joint reaction forces in drop landings. J. Biomech. 39, 119-124. doi:10.1016/j.jbiomech.2004.10.036

Pai, S., Ledoux, W.R., 2010. The compressive mechanical properties of diabetic and nondiabetic plantar soft tissue. J. Biomech. 43, 1754-1760. doi:10.1016/j.jbiomech.2010.02.021

Reilly, D.T., Burstein, A.H., 1975. The elastic and ultimate properties of compact bone tissue. J. Biomech. 8, 393-405. doi:10.1016/0021-9290(75)90075-5

Riemer, R., Hsiao-Wecksler, E.T., Zhang, X., 2008. Uncertainties in inverse dynamics solutions: A comprehensive analysis and an application to gait. Gait Posture 27, 578-588. doi:10.1016/j.gaitpost.2007.07.012

Roberts, T.J., Marsh, R.L., Weyand, P.G., Taylor, C.R., 1997. Muscular Force in Running Turkeys: The Economy of Minimizing Work. Science 275, 1113-1115. doi:10.1126/science.275.5303.1113

Schache, A.G., Blanch, P.D., Dorn, T.W., Brown, N.A.T., Rosemond, D., Pandy, M.G., 2011. Effect of running speed on lower limb joint kinetics. Med. Sci. Sports Exerc. 43, 12601271. doi:10.1249/MSS.0b013e3182084929

Schmitt, S., Günther, M., 2011. Human leg impact: energy dissipation of wobbling masses. Arch. Appl. Mech. 81, 887-897. doi:10.1007/s00419-010-0458-z

Skinner, N.E., Zelik, K.E., Kuo, A.D., 2015. Subjective valuation of cushioning in a human drop landing task as quantified by trade-offs in mechanical work. J. Biomech. 48, 1887-1892. doi:10.1016/j.jbiomech.2015.04.029

Stefanyshyn, D.J., Nigg, B.M., 1997. Mechanical energy contribution of the metatarsophalangeal joint to running and sprinting. J. Biomech. 30, 1081-1085. doi:10.1016/S0021-9290(97)00081-X

Tung, K.D., Franz, J.R., Kram, R., 2014. A test of the metabolic cost of cushioning hypothesis during unshod and shod running. Med. Sci. Sports Exerc. 46, 324-329. doi:10.1249/MSS.0b013e3182a63b81

Wakeling, J.M., Nigg, B.M., 2001. Modification of soft tissue vibrations in the leg by muscular activity. J. Appl. Physiol. 90, 412-420.

Willems, P.A., Cavagna, G.A., Heglund, N.C., 1995. External, internal and total work in human locomotion. J. Exp. Biol. 198, 379-393.

Winter, D.A., 1983. Moments of force and mechanical power in jogging. J. Biomech. 16, 91-97. doi:10.1016/0021-9290(83)90050-7 
373 Zelik, K.E., Kuo, A.D., 2012. Mechanical Work as an Indirect Measure of Subjective Costs Influencing Human Movement. PLoS ONE 7, e31143. doi:10.1371/journal.pone.0031143

Zelik, K.E., Kuo, A.D., 2010. Human walking isn't all hard work: evidence of soft tissue contributions to energy dissipation and return. J. Exp. Biol. 213, 4257-4264. doi:10.1242/jeb.044297 
Fig. 1. Soft tissues whose deformations may store and return mechanical energy during running. The passive deformation of heel pads, plantar fascia, cartilage at joints, intervertebral discs, muscles (deformations separate from muscle contraction), skin, and even shoes may contribute.

Fig. 2. Mean joint angle, moment, and power trajectories over a stride at seven running speeds. Angles and moments shown are sagittal plane values, and powers shown include three-dimensional rotation and translation, averaged across subjects $(\mathrm{N}=8)$ at each point in the stride cycle (beginning and ending with same-side ground contact). Elbow, shoulder, and lumbosacral joints are shown on an expanded scale due to smaller power contributions. Angles $\left(^{\circ}\right)$ and moments $\left(\mathrm{N} \cdot \mathrm{m} \cdot \mathrm{kg}^{-1}\right)$ are defined as positive in extension. Dimensionless units (right-hand axes) are defined using body mass, leg length, and gravitational acceleration as base units.

Fig. 3. Power trajectories over a step (half a stride) for a representative subject running at $3.4 \mathrm{~m} \cdot \mathrm{s}^{-1}$. (A.) Joint Power, referring to the summed power of all measured joints, shown here along with stance leg's ankle, knee, and hip. (B.) Center of mass (COM) work rate plus Peripheral power equals Total power for the body. (C.) Soft Tissue power is the estimated as the difference between Total power and Joint Power. Power is shown in $\mathrm{W}$ (left-hand axes) and in dimensionless units (right-hand axes). Representative subject has body mass $71.2 \mathrm{~kg}$, leg length $0.94 \mathrm{~m}$.

Fig. 4. Soft-tissue power over a step (half a stride) for a representative subject. Soft tissue performs net negative work during a step, a majority of which occurs as the foot impacts the ground during the stance phase. The profile resembles a spring-mass-damper system, with a damped elastic rebound (about $10-$ $15 \%$ of stride).

Fig. 5. Average net work rates for center of mass (COM) work and Joint Work across running speeds. Average work rate, defined as net work per stride multiplied by stride frequency. Net COM work rate is close to zero at all running speeds, as expected during steady gait, while Joint Work rate is net positive at most speeds. Data shown were linearly fit with running speed $(\mathrm{N}=8)$.

Fig. 6. Trends in average positive and negative power vs. running speed $(\mathrm{N}=8)$. (A.) Average positive and negative work rates for ankle, knee, hip, and soft-tissue, the latter performing work comparable to the leg joints. (B.) Positive and negative work rates for four measures: center of mass (COM) work, Joint Power, Total work (sum of COM and Peripheral work), and Soft Tissue Work (Total minus Joint Power).

Table 1. Quantitative results for stride parameters and powers, from linear regressions against running speed. Slope and offset refer to regression coefficients ( $\pm 95 \%$ confidence interval, c.i.), reported with $\boldsymbol{R}$, significance (S) denoted by asterisk with criterion $P<0.05$. Dimensionless (d'less) quantities are computed using body mass, leg length, and gravitational acceleration as base units. 


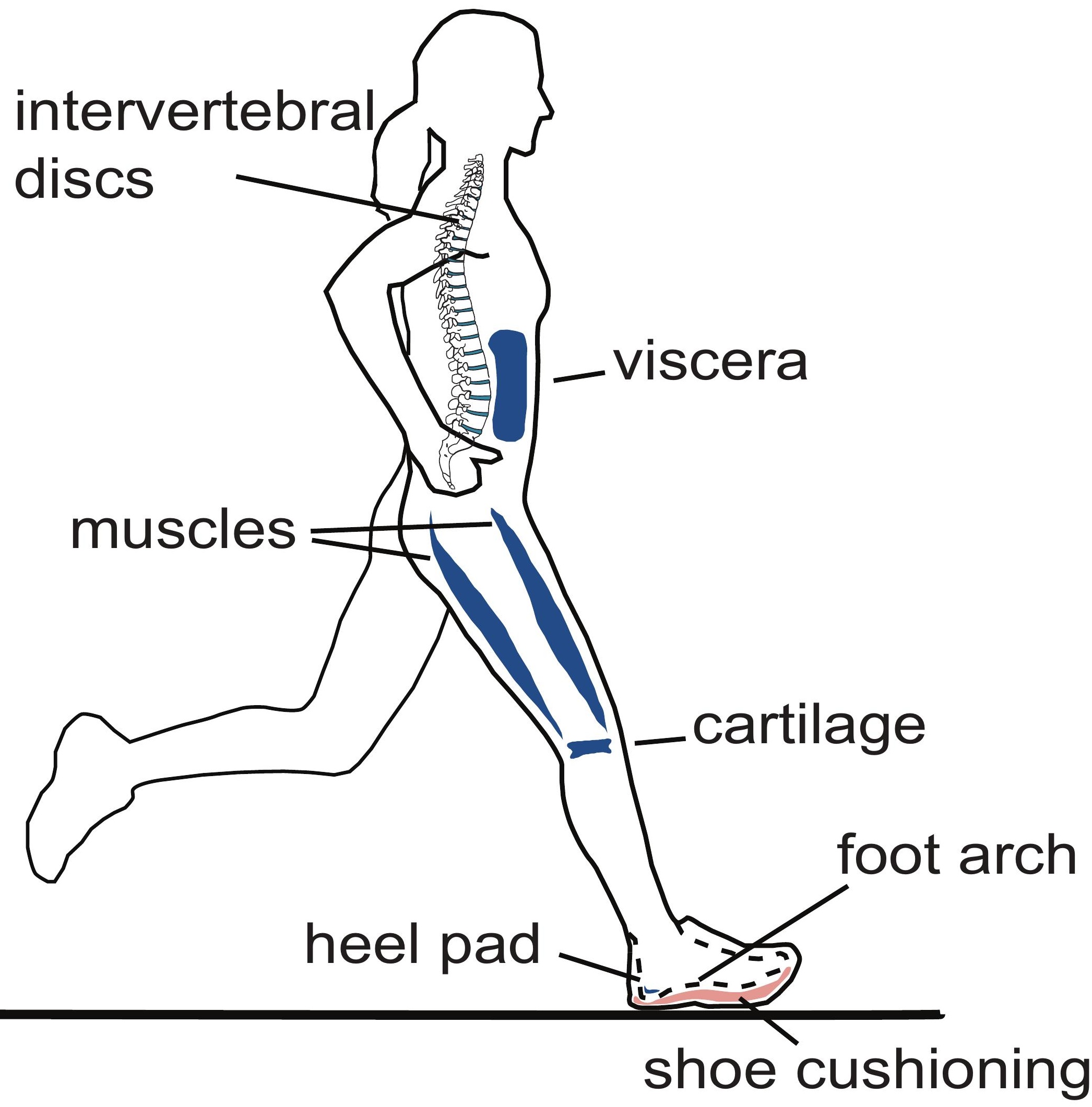

fiscs

heel pad 


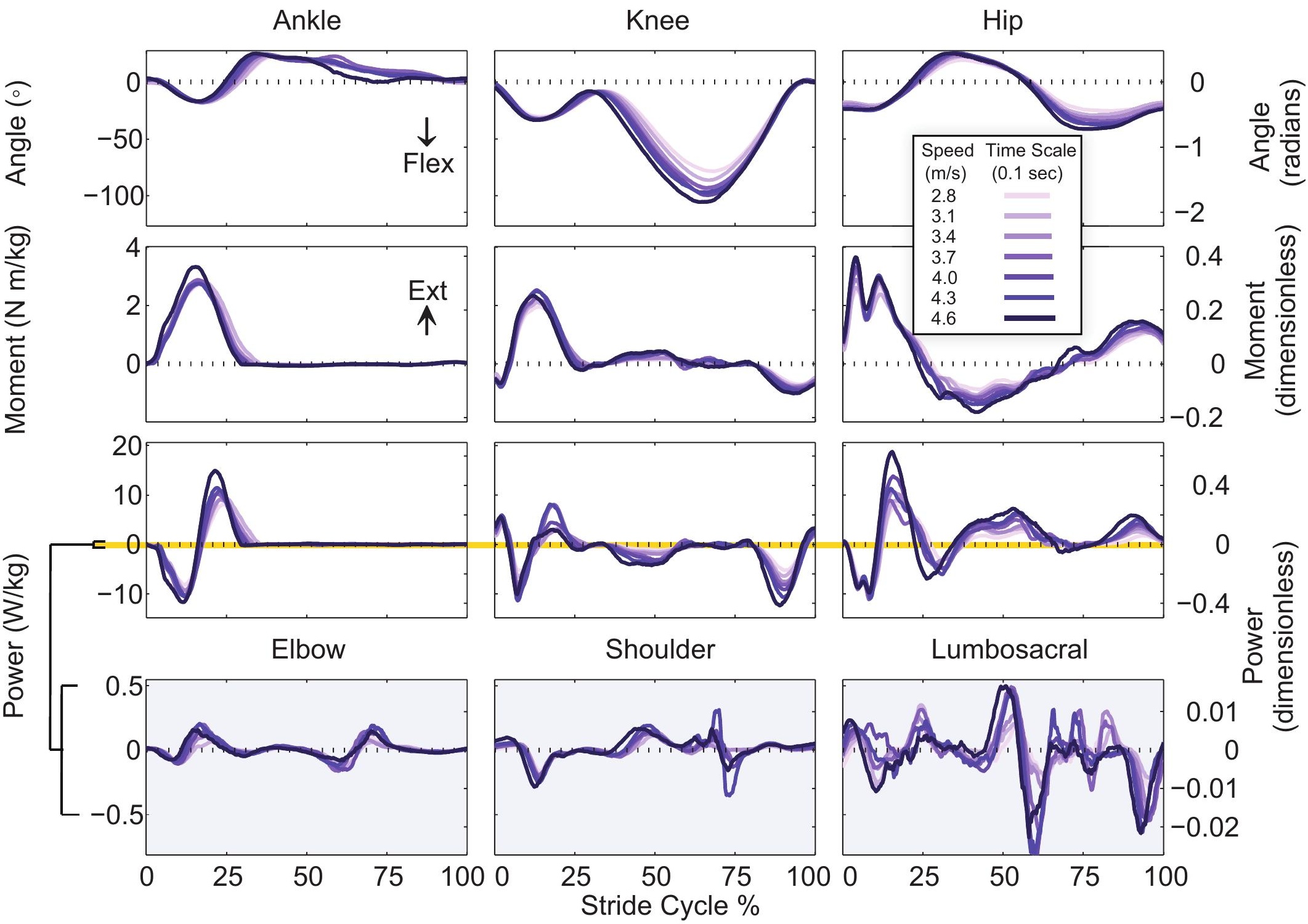




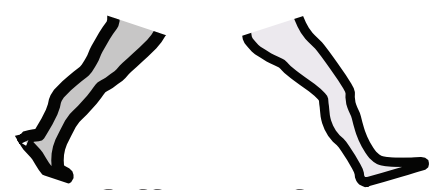

Right Heel Strike A Right Toe Off Left Heel Strike

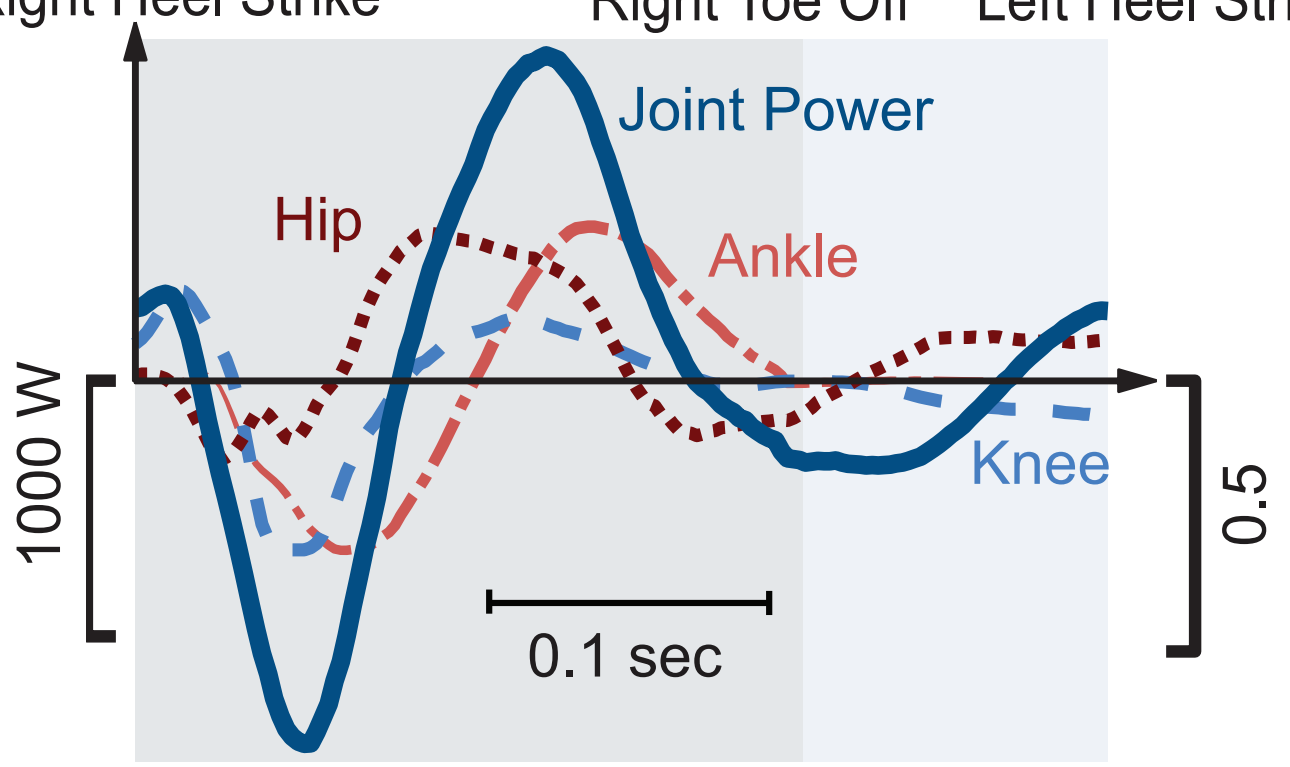

B

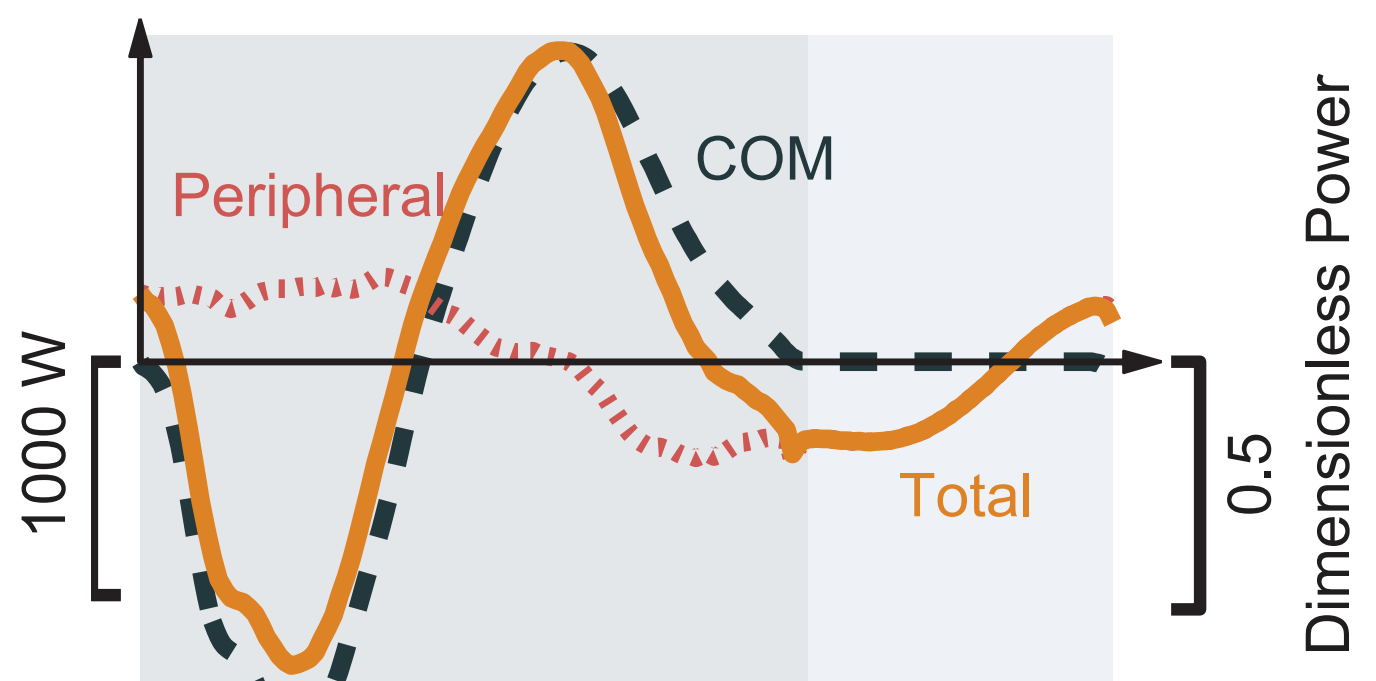

C
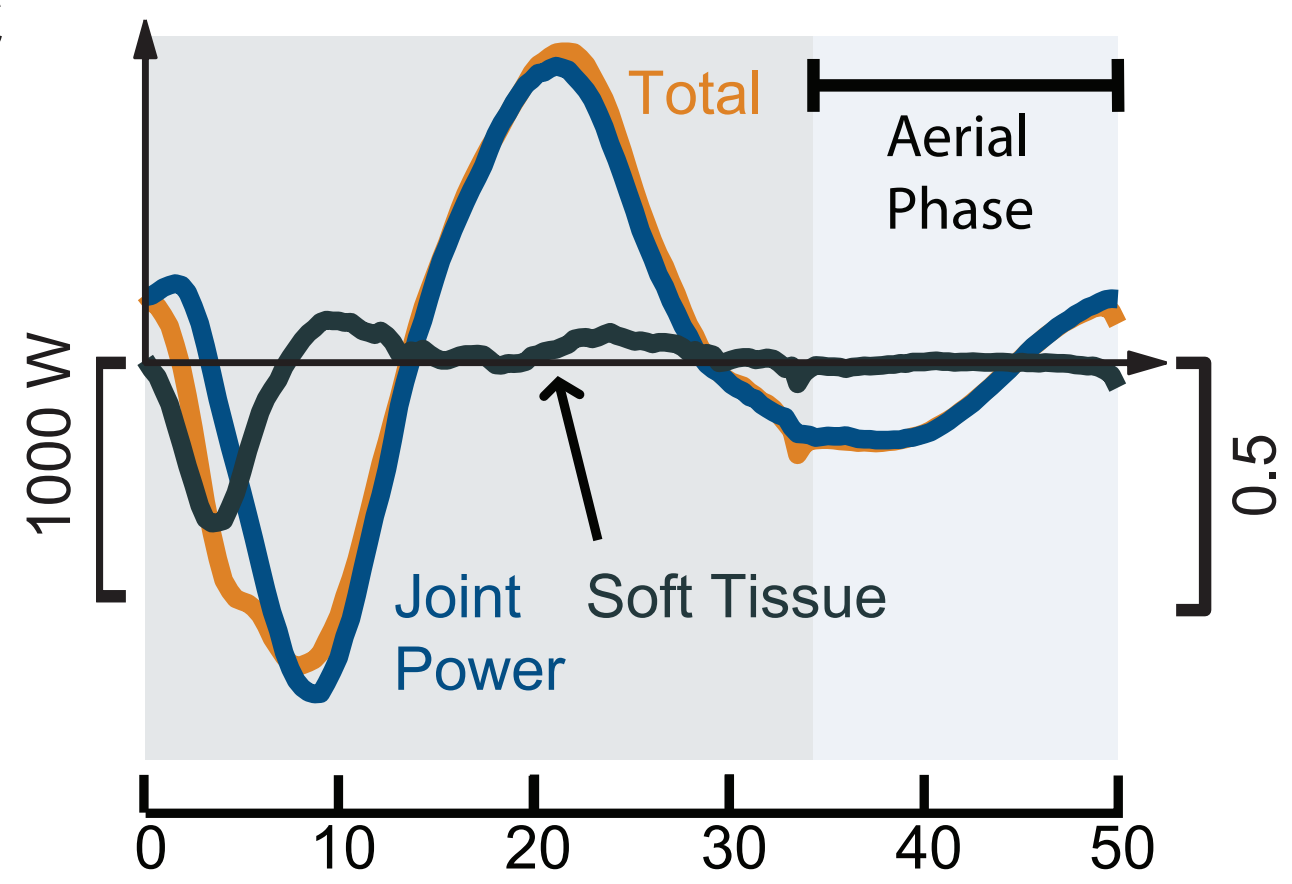

Stride Cycle (\%) 
Stride Cycle (\%)
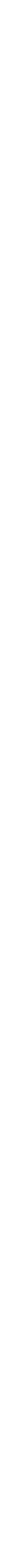

Figure 4 
Speed (dimensionless)

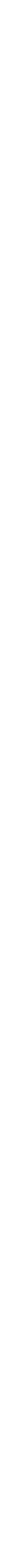

Figure 5 


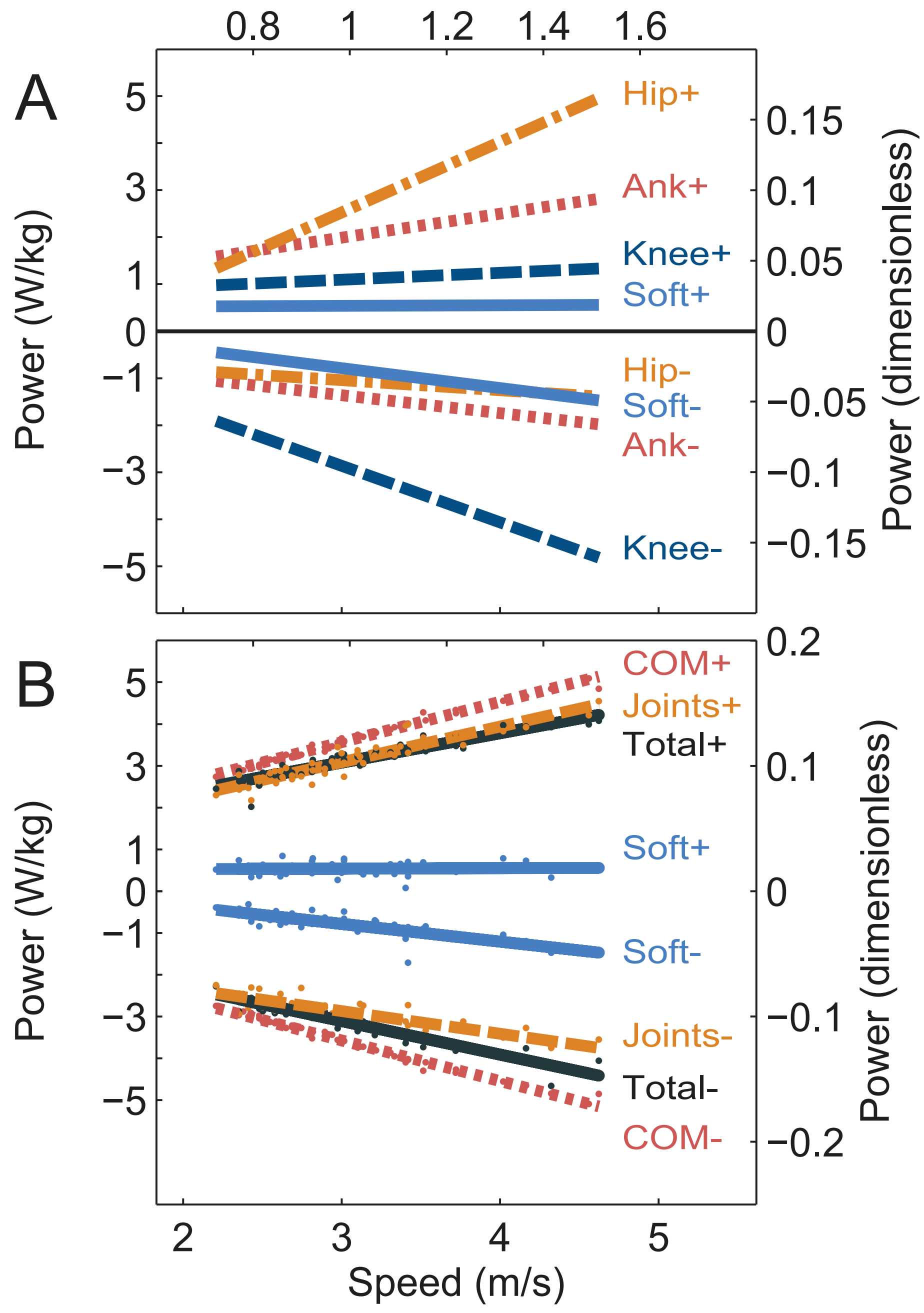

